\title{
Comment échapper à la honte du zamel : Vers la construction de la masculinité maghrébine queer
}

\author{
Daniel Maroun \\ University of Illinois at Urbana-Champaign
}

Le concept d'une identité maghrébine queer n'a d'autre expression que celle de la honte et de l'isolement social, surtout au Maroc. C'est dans ce contexte que l'écrivain et activiste social Abdellah Taïa vise à créer un espace littéraire qui confronte la dominance socio-culturelle de la patriarchie et la sexualité marginale des protagonistes au cœur de son œuvre. L'analyse 
de ses textes autofictionnels, L'Armée du salut, Une mélancolie arabe et Le Jour du Roi témoigne de l'émergence d'une identité marocaine queer. En tant qu'écrivain, Taïa met en valeur la construction, ou bien la reconstruction, d'un héritage marocain dépourvu d'une influence occidentale transgressive qui dépeignait le Maroc comme lieu où le colonisateur se rendait pour réaliser des activités sexuelles.

Vivant actuellement à Paris, Taïa écrit hors du pays qu'il aspire à changer, tout comme ses prédécesseurs Tahar Ben Jelloun et Abdellah Katibi (voir Morse). Selon Taïa, la production créative et critique de son pays ne peut se produire au Maroc, faute d'un lectorat marocain. Il ajoute: «Il y a un problème de lecture dans le monde arabe en général, et qui ne peut pas être dissocié de la crise politique que nous vivons, ni de la crise culturelle et identitaire, car tout est lié » (Sedrati, 2012). Pour l'écrivain, l'acte d'écrire supplée le manque sociosexuel dans la société marocaine.

À travers les trois ouvrages mentionnés plus haut, nous examinerons la construction d'une masculinité maghrébine queer au Maroc. Dans ce contexte, la masculinité est directement liée au patriarcat et donc, pour Taïa, l'affirmation d'une masculinité-queer oblige une déconstruction du patriarcat et des normes hétérosexuelles, car le queer est, par définition, ce qui est à part.

\section{Le patriarcat et le roi}

Taïa tente de démanteler le pouvoir du roi et donc, par extension, le patriarcat, afin de créer un espace et une expression littéraire de la masculinité queer marocaine. Dans 
Le Jour du Roi (2010), l'auteur parle de la famille, du père et de son roi, à qui tous les pouvoirs socioculturels appartiennent concernant la masculinité au Maroc. Le texte unit deux personnages, Khalid et Omar, qui partagent une amitié profonde et intime, malgré leurs différences socio-économique ${ }^{1}$. Pourtant, à la suite d'un concours remporté par-Khalid, lui donnant ainsi le droit de représenter l'école auprès du roi Hassan II, Omar se met à envier et à jalouser son camarade.

$\mathrm{Au}$ sein de ce texte est remise en question la performativité de la masculinité et des responsabilités familiales. Le père d'Omar n'incarne pas l'archétype du père consciencieux marocain qui sert de rôle socioculturel important. La mère d'Omar s'enfuit et son mari se retrouve complètement brisé puisqu'il ne peut plus remplir son devoir familial. Omar explique que son père « était l'âme de la famille. Le moteur de la famille. Le sang » (Taïa, 2010, p. 34). Le père est un repère culturel et idéal pour la famille marocaine. Cette citation met en lumière la façon dont Omar perçoit son père comme élément central de la famille, auquel tous les membres sont liés. La déchéance du père permettrait ainsi l'exploration de la déchéance d'une masculinité queer hors des normes marocaines.

C'est donc après le départ de la mère que le père abandonne son rôle de chef de famille pour retrouver son ancienne femme qui l'a quitté : «On disait dans le quartier qu'elle avait jeté un sort puissant à mon père. Un sort préparé par le sorcier le plus puissant de la ville. Du Maroc peut-être. »

\footnotetext{
${ }^{1}$ « J'ai éteint la lampe, et je l'ai rejoint dans le petit lit vert. Cela ne l'a pas réveillé. Il avait l’habitude. De moi. De mon corps. Deux. Un. » (p. 44)
} 
(Taïa, 2010 p. 36) Le père sollicite les conseils du sorcier le plus puissant du Maroc, Bouhaydoura, espérant rompre le charme qui, croit-il, l'a condamné. La discussion entre ces deux personnages illustre la pensée normative avec laquelle le père perçoit le monde quand ce dernier explique : «Un homme n'est rien, un homme est vide, nu, risible, sans une femme. » (p. 53) Est ainsi établi un paradigme hétéronormatif qui ne correspond pas à la réalité du texte, car ce dernier met en avant un décalage avec la famille traditionnelle marocaine.

Le père continue son monologue : « Elle est à moi. Elle est moi. J'aime qu'elle ne soit qu'à moi, qu'à moi...» (p.53). Il demande au sorcier : «Un homme, c'est ça, non? Non?» (p. 53), exprimant son désir de voir le paradigme normatif hétérosexuel réaffirmé. Il a raison de penser qu'au Maroc, la perception de la masculinité est intrinsèquement liée au mariage, puisque cette institution confirme et renforce la masculinité hégémonique (Smith, 2012, p. 36). Cette tentative de chercher l'approbation de Bouhaydoura est futile, car ce dernier lui réplique : "Vous avez tort» (p. 53), déclaration à laquelle le père ne s'attendait pas, répondant à Bouhaydoura : « Je ne comprends pas. » (p. 53) Ce qu'il ne comprend pas, c'est le «ça » («Un homme c'est ça non? ») qui n'existe plus dans la réalité du roman, étant donné que le texte construit un avenir où le père n'est plus le chef de la famille; son rôle familial disparaît dans ce texte où l'hétéronormativité est mise en question. C'est Omar, le fils, qui remplace le père quand celui-ci dit: "Aujourd'hui, c'est moi l'homme. Un homme pour mon père. » (Taïa, 2010, p. 35). En affirmant "pour mon père», Omar supplante son père et devient un «homme » à son tour, à sa manière. En remplaçant le père, il "queerise » le modèle familial marocain, créant un espace et une place pour une sexualité queer puisqu'Omar se 
substitue à la patriarchie et, par extension, à l'hétéronormativité. La réponse de Bouhaydoura («Vous avez tort») fait s'écrouler le monde du père tel qu'il le connaît et affaiblit la légitimité des rôles normatifs des sexes dans le texte, car on ne peut présupposer qu'un homme n'est rien sans sa femme.

Omar est présent tout au long de cet échange. Il comprend maintenant les nouveaux changements sur le plan sociolittéraire de ce que c'est qu'être un « homme». Vers la fin de la visite, Omar se dit: "Je l'ai saisie enfin» (Taïa, 2010, p.56), ce qui le met en opposition du père qui n'avait pas compris la réponse de Bouhaydoura. Mais on se demande ce qu'Omar a saisi. Dans "Je l'ai saisie enfin», il explique au lecteur que «le» représente les changements socioculturels relatifs au pouvoir de la patriarchie. Omar comprend que l'on est à la fin d'un paradigme : « Nous étions à la fin. Dans la fin du monde tel que je l'avais connu. La fin de ma famille.» (Taïa, 2010, p. 57). En fait, c'est la fin de la famille dans ce texte qui permet de nouvelles itérations de la performativité du genre et des rôles des sexes. Quand Omar explique: "Je l'ai saisie enfin ", il reconnaît que, même si son père est d'avis qu'un homme se fait par la présence d'une femme, ce n'est plus cas.

Omar se distancie de son père, devenu perclus à la suite de son obsession envers son ancienne femme, et note: «Ce n'est pas un bon exemple pour moi, cette conduite. » (p. 31) Il est conscient du fait qu'il est supposé apprendre les normes sociosexuelles de son père, mais il est conscient aussi que son père n'est plus le chef de famille: «Il ne faut pas que je devienne comme lui [...]. Déchéance d'un homme. » Alors, avec Khalid, Omar poursuit la création d'une masculinité marocainequeer qui n'est pas basée sur la tradition, mais sur l'expérience. 
Le Jour du Roi annonce l'abandon de la patriarchie marocaine dès le premier chapitre, qui traite d'un rêve où Omar rencontre le roi Hassan II. L'interaction qui se déroule pendant le rêve souligne la façon dont la masculinité et la paternité sont en mutation dans le texte. En effet, le rêve sape l'autorité paternelle que le roi illustre en tant que père de tous les Marocains. Dans ce rêve, Omar a l'honneur de se présenter auprès du roi pour lui baiser la main ${ }^{2}$. Omar s'approche du roi et ce dernier lui demande : «Comment je m'appelle?». Omar lui répond: «Hassan II... Le roi Hassan II du Maroc» (Taïa, 2010, p. 10). En nommant le roi, Omar lui donne une présence et une importance textuelles. Pourtant, le roi reste insatisfait : «Il me dit: "Non. Mon nom de famille? Quel est mon nom de famille?" »

Le roi, en insistant pour que soit mentionné son nom de famille, est à la recherche d'une reconnaissance sociale, comme s'il avait peur que son pouvoir disparaisse. Omar ne peut rien dire : "Je suis toujours muet. » (Taïa, 2010, p. 11). Il cherche la réponse mais ne la trouve pas. Le roi exige alors : «Mon nom de famille? Vite, Vite... mon nom de famille? Vite j'ai dit... ». Cette ténacité se transforme en violence, qui naît du manque de reconnaissance familiale: «Il [le roi] s'est rapproché de moi. Ses deux mains sont autour de mon cou, qu'il serre de plus en plus fort. » Le roi n'a pas de famille et cherche à éliminer la menace que l'ignorance d'Omar représente à l'ordre symbolique.

Omar s'évanouit, mais une femme de la cour du roi le réveille et lui chuchote: «Va vers lui, va vers le Roi, c'est

\footnotetext{
2 « Baiser la main de Hassan II : c'est le rêve de presque tous le Marocains. Je suis devant ce rêve qui se réalise. » (Taïa, 2010, p. 16)
} 
comme ton père. C'est ton père. » (Taïa, 2010, p. 12) Puis les suivants du roi se mettent à rire, la violence tourne au comique. Le bruit désoriente Omar, qui leur hurle : « Non, il n'est pas mon père. Le roi n'est pas mon père.» (p.13) Cette déclaration montre une négation totale de la puissance symbolique du roi comme père de tous les Marocains. Si le roi n'a pas de famille littérale (nom de famille) et symbolique (son peuple), il perd toute souveraineté. Omar est alors capable de créer un espace littéraire pour une masculinité et une sexualité marginale qui se met au centre du texte plutôt qu'aux marges d'une société littéraire. Dans ce rêve, Omar mine le pouvoir symbolique du roi pour pouvoir explorer une sexualité queer dans le texte, car la présence du roi représente le pouvoir de l'hétéronormativité, mais à quel risque? Une femme le réveille en disant : «Plus tard, ton châtiment sera pire, pire que tout. » (p.14) La citation révèle une confrontation entre la réalité sociale actuelle et celle du texte.

En déclarant que le roi n'est pas son père, Omar met en danger sa culture et son identité sexuelle normative. À la fin de son rêve, le sol s'ouvre sous ses pieds, le plongeant dans une chute interminable accompagnée par la voix de la femme répétant : «Bye-bye... Tu n'as plus de père... Bye-bye... Tu n'as plus de Roi...» (Taïa, 2010, p. 22) En ignorant le nom de la famille du roi, Omar produit une réaction en chaîne qui menace sa nationalité et sa masculinité. Pendant sa chute, il se demande: «Qui suis-je?» Mais la voix recommence à crier «bye-bye » et cherche à annoncer l'avenir du texte ainsi que celui d'Omar, celui où on aura éliminé les liens entre la paternité et la sexualité pour promouvoir de nouvelles interprétations de la masculinité. En sapant l'autorité 
sociosexuelle et culturelle de la dominance des "pères » et du « Père » (le roi), le texte de Taïa produit un monde littéraire où Omar et son ami Khalid redéfinissent la marocanité et leur propre sexualité.

Le résultat, alors, est un espace littéraire où les deux protagonistes marocains explorent leur sexualité, pas de manière explicite, mais plutôt d'une manière qui justifie une identité marocaine queer. J'utilise ce terme dans sa forme la plus pure, c'est-à-dire pour signifier ce qui n'est pas de la norme sociale.

Sans rentrer dans les questions complexes de l'identité sexuelle, je voudrais d'abord préciser que l'espace que Taïa crée dans son texte permet une exploration des sexualités non traditionnelles. Taïa/Omar ne cherche pas à étiqueter sa sexualité dans ce texte ${ }^{3}$. En fait, c'est Omar qui formule des réflexions : "Je ne suis ni garçon, ni fille. Je suis dans le désir. » (Taïa, 2010, p.179) Il se détache ainsi d'une tradition marocaine qui qualifie les hommes hétérosexuels de " garçon » et les homosexuels de «fille». Ce qui compte pour Omar (et, plus tard, pour d'autres personnages dans les textes de Taïa), c'est d'être dans le désir, dans «le moment du désir » (p. 179), un espace transformatif où l'on cherche à récrire toute la sexualité marocaine.

\footnotetext{
${ }^{3}$ Et aussi dans Une mélancolie arabe et L'Armée du salut. Il faut prendre en compte que ces trois textes (y compris Le Jour du Roi) font partie d'une évolution intime de l'écrivain, qui a bien sûr changé depuis leur création. Je suis d'avis que Taïa évite les étiquettes "gay» dans ses premiers romans et aussi dans ses premières interviews. Jean Pierre Boulé, dans son essai «Writing selves as mourning and Vita Nova: Abdellah Taïa's Un pays pour mourir », explique la transition entre les textes autofictifs de Taïa et ceux qui viennent de paraître, Infidèles (2012) et Un pays pour mourir (2015).
} 
Omar réfléchit : « Je vais le précéder [le désir], l'annuler, l'exploser, le réécrire.» (p. 179), car le désir n'est plus défini selon le pouvoir du patriarcat, étant donné que le rêve d'Omar l'a aboli. La relation entre Omar et Khalid témoigne de la réécriture de la masculinité au Maroc.

\section{L'homme et le zamel}

La réécriture de la masculinité marocaine se développe dans Une mélancolie arabe, où le lecteur part en voyage à Hay-Salam, le quartier natal d'Abdellah, et à Salé, sa ville. Le titre du roman introduit une perspective intéressante concernant le concept de ła mélancolie. Alors que la nostalgie souligne l'envie d'un retour au passé, la mélancolie fait référence à un présent dépressif lié, peut-être, à un événement spécifique du passé. Le titre suscite alors une réponse préméditée du lecteur, une attente que le roman définira comme mélancolie marocaine. Ce lien est renforcé par le titre du premier chapitre, « Je me souviens », qui engage une comparaison immédiate entre l'antériorité et le présent du texte, une antériorité où une masculinité queer marocaine se trouvait aux marges de la société, voire n'existait pas du tout.

La juxtaposition entre passé / présent (Maroc normatif / Maroc queer) se renouvelle dès les premières lignes du texte alors que le narrateur déclare être dans sa "deuxième vie » (Taïa, 2008, p. 9), ce qui soulève la question de savoir ce qui s'est passé dans la première d'entre elles. Dès les premières pages, Taïa promulgue un changement paradigmatique, un changement qui permet l'expression d'une masculinité marocaine queer. Une mélancolie arabe, comme Le Jour du Roi, 
témoigne d'un conflit, voire d'une collision entre l'homophobie du Maroc et la tentative de mettre au centre du débat social les sexualités marginales. Abdellah et un de ses amis passent les nuits ensemble, se masturbant sans penser à ce que cela implique. Le père de son ami les découvre et chasse Abdellah de la maison. Celui-ci erre dans les rues, réfléchissant contemplativement : « Je ne suis pas comme les autres » (p. 13), un rappel textuel du traditionalisme marocain auquel il est toujours confronté.

Pendant qu'il flâne, il rencontre une bande d'adolescents qui permet une juxtaposition saisissante avec l'aliénation qu'il avait subie précédemment. Il dit: "J'étais comme eux, absolument comme eux » (Taïa, 2008, p. 14), et il se lie à ce groupe socialement exclu en participant à la nouiba: "On faisait la nouiba: chacun se donnait à l'autre. On baissait nos pantalons et on faisait l'amour en groupe. » (p. 14) Le mot arabe est mis en relief dans la page, rappelant au lecteur que cette cérémonie est propre à la culture marocaine et que la performativité de sa masculinité sera toujours fondée sur la

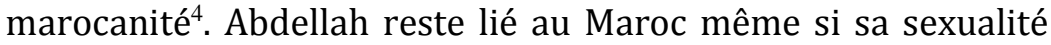
l'en éloigne.

Ce mot arabe n'est pas le seul à être employé dans le texte, car Taïa a tendance à utiliser zamel aussi plutôt que le terme péjoratif français «pédé ». Le protagoniste raconte : «Je restais avec eux même quand ils m'insultaient, me traitaient d'efféminé, de zamel » (Taïa, 2006, p. 14), ce qui montre qu'il

\footnotetext{
${ }^{4}$ La nouiba représente un égalitarisme sexuel étant donné que tous les participants pénètrent et sont pénétrés. Pour une étude profonde de cette cérémonie. Voir Hayes (2001) pour l'analyse de Messaouda d'Abdelhak Serhane.
} 
reconnaît dans sa situation sociosexuelle celle d'un paria. Cette tendance à utiliser le mot zamel remonte au fait que Taïa explore ce qui est propre à sa culture et ne peut pas être dit en français. En fait, il évite les mots occidentaux comme "gay», «homosexuel» ou «pédé » et préfère employer la circonlocution «l'amour interdit» (Taïa, 2008, p. 110) pour bien représenter sa culture dans cette langue. Bien sûr, certains critiques affirment que ce qui est en jeu est une masculinité " gay », « the process of asserting a gay identity in the context of a society where the existence of such an identity is often denied » (Smith, 2012, p. 35), surtout pour Taïa ${ }^{5}$. Mais plutôt que d'imposer un terme qui porte une identité, Taïa résiste.

Le terme nouiba est crucial parce qu'il désigne une cérémonie où les participants sont actifs et passifs. Il est important de le noter, car on ne questionne jamais la masculinité de celui qui pénètre, seulement celle de celui qui est pénétré6. Sophie Smith explique: «A normative masulinity displayed through penetration of the socially inferior Other in order to express its dominance, representing the physical manifestation of a rigid dichotomy whereby the penetrated is relegated to the status of non-man or, at best, 'less-of-a-man' » (p. 37) ${ }^{7}$. La cérémonie efface alors toute hiérarchie sexuelle, permettant aux membres de jouer tous les rôles. Smith souligne le fait que, dans les pays arabes, l'important, c'est l'acte sexuel

\footnotetext{
5 Je traduis : « le processus d'affirmation d'une identité gay dans le contexte d'une société où l'existence d'une telle identité est souvent refusée. »

${ }^{6}$ Voir L'Amour circoncis d'Abdelhak Serhane.

${ }^{7}$ Je traduis : «La masculinité normative, montrée à travers la pénétration de l'Autre inférieur au niveau social, pour exprimer sa domination et représenter la manifestation physique d'une dichotomie rigide où le pénétré est relégué au statut de non-homme ou, au mieux, de "moins-qu'un-homme". »
} 
plutôt que les personnes impliquées dans l'acte; alors, désirer un homme n'est pas problématique dans la culture musulmane (p. 38).

Taïa essaie de mettre au centre de la discussion sociale marocaine une nouvelle expression de la masculinité qui n'est pas hétérosexuelle et reléguée au terme zamel. Abdellah, le protagoniste, cherche à construire sa propre «véritable identité » (Taïa, 2008, p. 17), qui transgresse la connaissance socioculturelle du Maroc qui l'avait désigné comme zamel. Cette transgression est révélée quand Abdellah est harcelé par un garçon du quartier, Chouaïb, qui veut le violer. Face aux insultes et aux stigmates de la féminisation de la part de Chouaïb (qui l'appelle « Leïla »), Abdellah se livre à un monologue :

J'ai voulu un moment lui donner mon vrai prénom, lui dire que j'étais garçon, un homme comme lui... Lui dire qu'il me plaisait et qu'il n'y avait pas besoin de violence entre nous, que je me donnerais à lui heureux si seulement il arrêtait de me féminiser... Je n'étais ni Leïla, ni sa sœur, ni sa mère. J'étais Abdellah, Abdellah du Bloc 15 et dans quelques jours j'allais avoir 13 ans. (Taïa, 2008, p. 21)

Abdellah choisit de rompre la classification constante de «Leïla » de Chouaïb (et par là avec la société marocaine) en déclarant sa propre identité, qui implique sa masculinité queer.

Quand Abdellah déclare "un homme comme lui », il se sépare de la tradition marocaine, laquelle traite l'homme passif de zamel. Il explique : "Je voulais lui dire et redire qu'un garçon est un garçon, et une fille est une fille » (Taïa, 2008, p. 22), ce qui paraît essentialiste. Il continue ainsi : «Ce n'était pas parce que j'aimais sincèrement et pour toujours les hommes qu'il pouvait se permettre de me confondre avec l'autre sexe", admettant que les hommes peuvent aimer les hommes. Il 
reprend son identité en disant: "J'étais Abdellah, Abdellah du Bloc 15 », se donnant une place dans la société marocaine. Il craint cette étiquette de "Leïla», car il craint de perdre son identité en tant qu'homme et Marocain. Pourtant, lorsqu'il annonce « J'étais Abdellah », il refuse l'identité que la société lui impose. Pour Chouaïb, Abdellah doit être «Leïla » parce qu'un homme comme Abdellah qui se laisse pénétrer n'existe pas (Smith, 2012, p. 44). Seules les femmes se font pénétrer.

Abdellah note que la féminisation de sa personne nie son individualité : elle détruit« mon identité, mon histoire » (Taïa, 2008, p. 21), dit-il, évoquant un passé où il a déjà justifié sa masculinité. «Leïla» remet en cause la présence de la masculinité d'Abdellah. Il s'en prend à Chouaïb: «Je suis Abdellah... Abdellah Taïa » (p. 24). En se nommant, en donnant son nom de famille, qu'il a d'ailleurs nié au roi Hassan II, il se révolte contre le standard culturel que la société lui impose. Il se donne un héritage, une famille, une place dans la culture marocaine en disant « Taïa ».

Chouaïb se trouve stupéfait. C'est Abdellah qui remarque : "Il était surpris, dans mes yeux, il lisait enfin autre chose que la peur et la soumission. » (Taïa, 2008, p. 24) L'« autre chose » que lisait Chouaïb est la reconnaissance de la masculinité marocaine queer qu'il avait essayé de supprimer. Ce qu'explique Abdellah souligne à la fois le choc et la compréhension que la masculinité marocaine peut avoir une expression ou une version queer sans être reléguée au féminin, niant son existence sur le champ socioculturel.

Le texte de Taïa établit une forme de la masculinité marocaine à travers une transmission, soit littéralement dans 
l'acte sexuel ou, de manière métaphorique, à travers les transmissions entre "générations » ou itérations de soi ${ }^{8}$. Cette première partie du roman est intitulée "Je me souviens », mais une fois que Taïa apprend à réclamer son identité ("Je suis Abdellah Taïa ») et que Chouaïb le reconnaît (« Il lisait enfin autre chose»), le chapitre se termine. Maintenant, le protagoniste ne se souvient plus, il raconte l'actuel où il met au centre sa performance d'une masculinité queer à la marocaine.

En fait, la première partie du texte « se souvient» de ce qui était impossible, c'est-à-dire d'une masculinité queer marocaine positionnée contre une masculinité dominante, traditionnelle, où existe la honte du zamel. Pourtant, cet acte de parole contre Chouaïb met en question l'impossibilité d'une masculinité queer marocaine et crée un espace littéraire pour sa propre interprétation de la masculinité marocaine. Intitulée "J'y vais », la deuxième partie place cette masculinité au cœur du roman, et place le discours hétéronormatif à la périphérie, même si la communauté qui entoure le protagoniste reste fortement ancrée dans ses traditions et normes. C'est là qu'Abdellah mène le lecteur, il va au nouveau centre du texte, et on y va aussi.

La construction de la masculinité marocaine, chez Taïa, est encore mieux explorée dans L'Armée du salut (2006). Les rapports familiaux, dans ce texte, permettent à Abdellah (le protagoniste) d'utiliser sa famille afin de construire davantage son concept d'une masculinité queer marocaine. Sa conscience sexuelle est un produit des rapports de famille appris dès

\footnotetext{
${ }^{8}$ La transmission intergénérationelle fait écho au texte épistolaire publié dans Lettres à un jeune marocain, où Taïa écrit à son neveu à propos de la mort du père de Taïa et de la culpabilité que ressent l'auteur concernant cette mort.
} 
l'enfance : «Dans ma tête, la réalité de notre famille a un très fort goût sexuel, c'est comme si nous avions tous été des partenaires les uns pour les autres. » (Taïa, 2006, p. 15) La nature incestueuse de cette citation fait écho au concept, explicité ci-dessus, selon lequel la masculinité chez Taïa est conçue à travers un échange ou une transmission qui déclenche une réaction ou, plutôt, la réalisation d'une identité.

L'Armée du salut est à part des autres œuvres de Taïa parce qu'il sexualise le protagoniste, Abdellah, dès les premières pages. Le "goût sexuel » qu'il remarque souligne qu'Abdellah était toujours au compte d'une sexualité, mais qu'il ne l'avait peut-être pas encore construite. Cette sexualité, qui lui permet de recréer sa masculinité « non-normative » selon sa culture, est passive, inquisitive, une source de connaissance et pas de honte comme celle du zamel: «Si la sexualité est omniprésente, elle n'est pas culpabilisante.» (Parris, 2009, p. 661) Dans ce texte, elle est encourageante.

Le frère d'Abdellah représente la masculinité traditionnelle marocaine et, partant, ce personnage joue un rôle important dans la formation de cette masculinité queer qu'Abdellah manifeste. Son frère symbolise plus qu'une figure fraternelle; il supplante le père sur le plan de l'éducation socioculturelle et intergénérationelle: «Il est plus que mon frère.» (Taïa, 2006, p. 33) Ce symbole fraternel évoque un concept statique de la masculinité et de la normativité que peut étudier son petit frère : "Mon frère est là depuis le début » (p. 33) et donc en dévier. Le frère représente un point de repère culturel et masculin pour Abdellah : «Il est l'homme grand que je voudrais être un jour.» (p. 33). Des critiques comme David Parris soulignent l'importance que joue Abdelkébir dans la 
construction d'une masculinité queer marocaine pour Abdellah : "Le rapport avec le grand frère est un élément-clé dans l'identité masculine du narrateur » (2009, p. 662). Mais à quel niveau ou bien comment cet élément-clé opère-t-il dans l'univers queer d'Abdellah. qui est toujours en négociations?

Le lecteur comprend qu'Abdellah ne va ni grandir comme Abdelkébir, ni assumer les mêmes rôles sexuels que son frère parce qu'il n'y a pas encore de masculinité queer marocaine. Le protagoniste se trouve dans la chambre de son frère : «En son absence j'entrais par la fenêtre dans sa chambre [...]. Je baignais dans l'odeur forte d'Abdelkébir, son odeur d'homme. » (Taïa, 2006, p.35) Pour créer cette masculinité queer, Abdellah consomme le paradigme masculin que représente son frère et queerise ainsi l'idéal marocain masculin. Dans cette chambre, Abdellah établit une ambiance d'homme qui entoure le lecteur (« odeur d'homme »), là il découvre des vêtements sales :

Sous sa bibliothèque il cachait des slips qui avait une odeur particulière et étaient tachés de blanc à l'intérieur. Je mis du temps avant de comprendre. C'était son sperme. (p. 35)

C'est ici que le queer se confronte au normatif, car Abdellah ne sépare plus les deux: «Moi, même le sperme de mon frère je le connaissais. Je le touchais, je l'étudiais, je le reniflais [...]. Ce sperme venait de lui. Il était lui.» Le sperme d'Abdelkébir symbolise le corps normatif hétérosexuel masculin par lequel Abdellah est obsédé. Il envisage de le manger, de l'avaler pour pouvoir créer une masculinité queer à la marocaine, basée sur son frère, le plus grand représentant des normes marocaines. Un tel désir et une telle action rassemblent le monde normatif auquel appartient Abdelkébir et le monde queer que construit Abdellah. 
Abdelkébir finit par se marier, une action qu'Abdellah perçoit comme une trahison contre le monde queer : «C'était une trahison, non de sa part, mais de la part de la société. » (Taïa, 2006, p. 37). Abdellah souligne comment la société oblige son frère à se marier et force l'individu queer à se situer aux marges de la société, tout comme son rapport avec son frère est subverti par l'obligation culturelle du mariage. Le mariage n'existe pas dans le monde queer d'Abdellah; Abdelkébir ne peut donc y exister: «Abdelkébir n'était plus mon frère d'avant», le frère dont la présence lui avait permis de construire une masculinité qui lui appartenait.

\section{Conclusion}

Dans ces trois textes, Abdellah Taïa cherche à créer un espace où l'expression d'une masculinité queer marocaine peut avoir une voix, voire une performance sans être reléguée au féminin. La confrontation entre les normes masculines marocaines et les nouveaux changements permettent à Taïa de ne pas trahir sa culture marocaine, mais surtout établit une masculinité queer basée sur sa culturelle natale, qui lui permet de se donner une place égale parmi toutes les autres expressions de la masculinité. Dans Le Jour du Roi, l'influence patriarcale est affaiblie, menacée et chassée du texte par l'amour entre deux jeunes adolescents. Une mélancolie arabe et L'Armée du salut contribuent à une réaffirmation (queer) d'une identité et d'une masculinité face aux insultes et aux étiquettes de zamel, un terme qui s'oppose à la masculinité. Ces textes de Taïa mettent en lumière le queer tout en étant conscients des impacts sociaux des coutumes et lois marocaines. Le processus n'est pas 
complet. Le protagoniste de L'Armée du salut commente: "Je me trompais. J'allais évoluer longtemps encore. » (Taïa, 2006, p.153) Taïa, en tant qu'écrivain, tente de réconcilier sa masculinité avec la culture marocaine et l'islam.

\section{Bibliographie}

Boule, Jean-Pierre. (2016), «Writing selves as mourning and Vita Nova: Abdellah Taïa's Un pays pour mourir», Contemporary French Civilization, vol. 41, ํㅡ 1.

HAYES, Jarrod. (2001), «Queer Resistance to (Neo)-colonialism in Algeria », dans John C Hawley (ed.), Postcolonial, Queer: Theoretical Intersections, Albany, State University of New York Press, p. 79-96

MorSE, Erik. (2009), «Expat Lit: Abdellah Taïa » Interview, 5 novembre, $<$ http://www.interviewmagazine.com/culture/abdellahtaia\# $>$.

PARRIS, David L. (2009), "Amours "inter-dites": allers (et retours) Maroc-France », International Journal of Francophone Studies, vol. 12, no 4, p. 665-670.

SEDRATI, Brahim. (2012), «Interview croisée : Deux Marocains à Paris [Tahar Ben Jelloun et Abdellah Taïa]", TelQuel, 25 octobre, <http://telquel.ma/2012/10/25/interviewcroisee-deux-marocains-a-paris $5414723>$.

SERHANE, Abdelhak. (1995), L'Amour circoncis, Casablanca, Éditions Eddif.

SMITH, Sophie Catherine. (2012), «Être ce qui ne se dit pas: negotiating a gay identity in Abdellah Taïa's Une mélancolie 
arabe », International Journal of Francophone Studies, vol. 15, no 1 p. 35-51.

TAÏA, Abdellah. (2010), Le Jour du roi, Paris, Seuil.

—. (2009), Lettres à un jeune marocain, Paris, Seuil.

—. (2008), Une mélancolie arabe, Paris, Seuil.

—. (2006), L'Armée du salut, Paris, Seuil.

\section{Résumé}

À travers une étude de trois ouvrages, Le Jour $d u$ Roi, Une mélancolie arabe et L'Armée du salut d'Abdellah Taïa, j'examine la construction d'une masculinité maghrébine queer au Maroc, qui n'avait guère d'autre expression que celle du zamel, terme péjoratif de l'arabe qui se rapporte à un homme aimant se faire pénétrer par d'autres hommes. Le zamel est la honte d'une société hétéro- et islamo-centrique dont les normes sont construites à partir de la soumission au patriarcat. Pourtant, cet article explorera comment Taïa récupère une masculinité queer marocaine auprès de la féminisation coutumière que subit cette population périphérique. Afin d'affirmer l'existence d'une masculinité queer dans ces trois textes, je propose une lecture où l'écrivain déconstruit le pouvoir du patriarcat, dont la chute permet de nouvelles expressions hors des normes marocaines. En effaçant la dominance sociosexuelle et culturelle du roi et, par-delà, de la paternité comme concept social dans Le Jour $d u$ Roi, Taïa construit un monde littéraire qui permet une réécriture de l'expression de la marocanité, en particulier l'expression d'une masculinité queer marocaine. Cette lecture possible apparaît également dans Une mélancolie arabe et dans L'Armée du salut, où le narrateur-protagoniste réaffirme son existence et sa masculinité face aux confrontations d'une société qui les qualifie injurieusement de zamel. Par l'ensemble 
de ses textes, Taïa cherche à se bâtir une identité masculine marocaine qui est queer dans une société où celle-ci n'a pas d'expression. Ces textes servent d'espace de confrontation entre l'islamonormativité et sa sexualité.

\section{Abstract}

Through the examination of Abdellah Taïa's three textes, Le Jour du roi, Une mélancolie arabe et L'Armée du salut, we will look at the construction of a queer-Maghrebi masculinity that previously had no other expression aside from zamel, a derogatory Arabic term designating a man who prefers being penetrated by other men. The zamel represents the cultural shame of a hetero- and islamo-centric society whose norms are built upon the rule of the patriarchy. This essay will explore, however, how Taïa reestablish a queer Moroccan masculinity for a peripheral population who is customarily feminized. In order to confirm the existence of a queer masculinity in these three texts, we propose a reading where the author deconstructs the power of the patriarchy whose downfall permits new expressions of masculinity outside of Moroccan norms. By erasing the sociocultural and sexual dominance of the king, and by extension, of paternity as a social construct, Taïa constructs a literary world in Le Jour du Roi that allows a rewriting of how Morocanness is expressed, and in particular, how a queer, Moroccan masculinity is expressed. This reading appears equally in Une mélancolie arabe and L'Armée du salut where the protagonist-narrator of each text reaffirms his existence and his masculinity while at the same time being confronted by a society that qualifies them as zamel. Through this collection of texts, Taiia constructs a Moroccan masculinity that is queer in a society where such an expression does not exist. These texts serve as a confrontational space between islamonormativity and sexuality. 